\title{
8. GENERAL REMARKS ON ORBIT AND EPHEMERIS COMPUTATION
}

\author{
B. G. MARSDEN \\ Smithsonian Astrophysical Observatory, Cambridge, Mass., U.S.A.
}

\begin{abstract}
Present and future requirements concerning ephemeris prediction for comets (particularly the short-period comets) are discussed, with reference to both positions and magnitudes.
\end{abstract}

The recoveries of periodic comets at their various returns to perihelion are usually made with long-focus reflecting telescopes. Time on such instruments is at a premium, however, and since the usable fields are so small, it is very helpful if the positions predicted for the comets can be accurate to $5^{\prime}$ or better (Roemer, 1968). Of course, there are special problems with comets that have previously made only one appearance, or that have not been observed for several decades, or that experience large, irregular nongravitational anomalies in their motions - a particularly troublesome situation because it cannot be anticipated - but in general to obtain an accuracy of $5^{\prime}$ is not really asking for very much. It is perhaps not widely appreciated that, except in 1855, P/Encke was always recovered within $3^{\prime}$ of Encke's predicted positions. The same accuracy was achieved with Möller's prediction for the fourth apparition of $\mathrm{P} / \mathrm{Faye}$ and with Leveau's prediction for the third apparition of $\mathrm{P} / \mathrm{d}$ 'Arrest, the latter in spite of that comet's having been missed at its previous return and being strongly perturbed by Jupiter. All this was accomplished a century ago and more! There are of course several examples in more recent times where similar accuracies have been achieved using logarithms or desk calculators.

But there are a good many other instances where observers have had to waste time over predictions that are considerably in error as the result of careless computations, even when high-speed automatic machines have been utilized. It is certainly desirable to try to allow for the nongravitational effects in some way. However, to obtain predictions accurate to $5^{\prime}$ it is certainly not essential to have a sophisticated computer program to determine these effects. If a comet has a revolution period of less than $20 \mathrm{yr}$ and has been observed at its two most recent returns, it is merely necessary to verify that the perturbations - those by Jupiter and Saturn, at any rate have been calculated correctly, both between the two recent apparitions and afterwards. One need not try to obtain a least-squares fit to the observations: just check that the residuals are reasonably small. The next predicted perihelion time will then in general be good to 0.1 day, for there are few such comets where the nongravitational effects over one revolution amount to more than this; and unless the comet comes close to the Earth, the predicted ephemeris should easily be within $5^{\prime}$ of the truth. It may even be possible to refine the prediction by applying to the perihelion time the nongravitational correction $\Delta P$ (Marsden, 1972, Table I).

If a comet has been missed at some of its recent returns, either since its latest apparition, or between its two latest apparitions, or both, one can still often carry 
out this procedure with success, applying to the perihelion time instead the correction

$$
\Delta T=\frac{1}{2} n(n+N) \Delta P,
$$

where $n$ is the number of revolutions since the last apparition and $N$ the number of revolutions between the last two apparitions. Of course, this procedure becomes less reliable when $n$ or $N$ is large or if there have been close approaches to Jupiter.

In any case, the orbits of the short-period comets are continually requiring attention, now as much as ever before. Because of the nongravitational effects, we shall probably never arrive at the situation we have with most of the minor planets, where ephemerides can be predicted half a century ahead and the objects easily recovered then. With new short-period comets still being discovered at the rate of seven per decade, with a larger proportion being successfully recovered at subsequent returns, and with several of the comets given up long ago as lost also being recovered, the amount of necessary computational work is rapidly increasing. There is a need for greater cooperation among those in situations where they are able to provide satisfactory predictions for periodic comets. Duplication of effort has in the past been stressed as important. Perhaps some duplication is still desirable; but if the predictions are satisfactory, it should no longer be necessary, and efforts can be put toward assuring that reliable computations are made on more comets. Fortunately, we do nowadays have the high-speed computers, and the coordinates of the perturbing planets can be easily read from magnetic tape or directly generated by the computers. Some progress has been made with putting cometary observations into machinereadable form: we have at the Smithsonian Astrophysical Observatory nearly 6000 positions of short-period comets (as well as 4000 positions of long-period comets) on punched cards; for 38 of the 62 comets of more than one appearance we have on cards observations made at all the apparitions since discovery, and there are only 14 cases where we do not have observations at every apparition since 1925. All the observations have been precessed to equinox 1950.0, the file is kept up to date and is in fact complete for all comets that have appeared since the beginning of 1965 except that many observations known to be erroneous have been removed.

As for the long-period, single-apparition comets the principal computational effort is in quickly obtaining orbits to yield reliable ephemerides. This phase of the work is nowadays conducted at least as satisfactorily as before. Since new comets are usually quite bright most of the astrometric observations are made with small to moderate wide-field instruments, and it is encouraging to note that there has of late been a considerable increase in the number of such observations made from the Southern Hemisphere. If a long-period comet remains under observation for at least four or five months, it is customary for a 'definitive' orbit to be calculated, in which all available observations are discussed and planetary perturbations taken into account. An important reason for doing this has been to verify whether comets originate in the solar system. The calculation of the 'original', and also the 'future', barycentric orbits for past comets is now simplified by means of the tabulation by Everhart and Raghavan (1970). But this work is not so important as previously, and because of 
nongravitational effects one certainly cannot regard the few original orbits found to be hyperbolic to several times their mean errors as evidence for interstellar origin: a strong reason for believing that we can indeed detect nongravitational effects in the motions of comets 1957 III and 1960 II (Marsden, 1972) is that allowance for them quite definitely makes the original orbits elliptical, and this was not the case when nongravitational terms were omitted. In future, the emphasis on the orbits of longperiod comets should perhaps be to ascertain whether nongravitational effects are more generally detectable.

One aspect of ephemeris calculation that continues to be unsatisfactory is magnitude prediction. For faint comets, particularly short-period comets whose recoveries are expected, ephemerides often give magnitudes that are far too bright. One must bear in mind again the fact that these comets are most likely to be observed photographically with long-focus reflectors, and further, that the observers would like to limit the exposure times so that they obtain cometary images that are suitable for astrometric measurement; ideally, the magnitude should be that of the nucleus. On the other hand, magnitudes so calculated are very misleading for comets that come bright enough to be seen in small telescopes; such magnitudes are discouraging, not only for amateurs, but also for professionals who wish to make physical observations of the comets.

Since there are observable at any given time a lot of very faint comets and not more than two or three bright comets it seems preferable to try basically to predict the 'nuclear' magnitudes. In a few cases an observer really comes close to observing a true nucleus, and one can use a magnitude formula that varies according to the inverse square of heliocentric distance (perhaps with a phase effect); more usually, however, what the observer reports is more consistent with an inverse fourth-power of variation with heliocentric distance, and occasionally even an inverse sixth-power law may be required. For bright comets one should try to predict the 'total' magnitude as well; indeed, for newly discovered comets nuclear estimates are rarely available. The total magnitude will almost certainly have a stronger dependence on heliocentric distance, and considering the nature of the comet one might reasonably choose an inverse fourth-, sixth- or eighth-power law. If both total and nuclear magnitudes are being predicted, there seems little point in listing sets of numbers differing only by a constant; one should use two different laws, and an individual observer can then select the one that suits him best, adjusting the constant as he desires. Whatever is done in practice, however, it should be clearly indicated whether 'total' or 'nuclear' magnitude is meant, and a convenient notation would be one based on the telegraphic code, using $m_{1}$ for total magnitudes and $m_{2}$ for nuclear magnitudes.

\section{References}

Everhart, E. and Raghavan, N.: 1970, Astron. J. 75, 258.

Marsden, B. G.: 1972, this Symposium, p. 135.

Roemer, E.: 1968, Trans. IAU 13B, 115. 